\title{
Research of EEG-Based Brain Fatigue Using Mutual Information
}

\author{
Yu Zhou ${ }^{1 *}$ \\ ${ }^{1}$ School of Electric Power, North China University of Water Resources and \\ Electric Power, Zhengzhou 450011, China \\ zhouyuchina@126.com
}

\begin{abstract}
In probable theory and informational theory, the mutual information of two random variables is quantity that measures the mutual dependence of the two random variables. The most common unit of measurement of mutual information is the bit, when logarithms to the base 2 are used. The brain is a complex system of collaborative work, and the brain information transfer need to be completed by numerous neuronal group collaboration. Analysis of synchronous relationship between multi-channel EEG signal can be obtained from different areas of the brain, informational integration, transmission and processing of the important information. Two channel synchronous analysis method is mainly used for analysis of the relationship between the two channel EEG signal, which can be divided into linear and nonlinear methods, and linear methods including correlation and consistency based on spectrum. The brain has obvious non-linear characteristic and dual channel, which are based on the nonlinear analysis of rapid development of synchronous method, for example, phase synchronization, nonlinear independence, mutual information and nonlinear correlation. The research content of this paper mainly is the extraction of EEG signals of normal and fatigue state, the EEG data denoising, filtering after pretreatment, combined with information entropy between information entropy is calculated for each channel EEG signals and any two channels of EEG signals, which can be get the mutual information matrix of each channel EEG the correlation analysis, the degree of signal of each channel height, and each channel under the change of EEG correlation and comparison of two state. The specific use of the MATLAB programming language is to achieve, and the results are analyzed in order to obtain the brain fatigue effects on brain network. By designing and running the program, the method of designing is verified.
\end{abstract}

Keywords: Brain Fatigue, Mutual Information, MATLAB

\section{Introduction}

Biological[1-8] phenomena is one of the basic characteristics of life activities, the electrical activity of organisms is various performance, to the whale, small to bacteria, has biological electricity or strong or weak. The study found that modern physiology, all organs of the human body would produce biological phenomena, and in the form of electricity, action potential, through the corresponding nerve fibers to excitatory transmission to the brain center, center of the brain in action potential way of nerve impulses signal through corresponding nerve fibers to the effector, functional activities resulting organ or organization. The cerebral cortex neuron with bio-electrical activity, often change the rhythmic potential continuously, called the spontaneous electrical activity of the brain. Guide the electrode will place on the scalp to record cortical brain waves, is known as EEG. Brain waves are the brain's activity, the formation of potential difference between the cerebral cortex cells, resulting in a current in the brain cortex of extracellular. It records the activities of the brain waves of change, which are the brain nerve cell electrophysiological activity in the cerebral cortex or the whole surface of the 
scalp to reflect. Mutual information is an information theory based on information entropy theory, the interactions can be from the angle of information transmission characterizations of functions in different brain regions. Widely used in EEG signal analysis of phase synchronization analysis, the utility model has the advantages of neglecting the effect of signal amplitude and phase relationship between the two study signal, which can detect the weak mutual contact. The information entropy of information theory is particularly suitable for studying the relationship between stimulus and response based on the method with the importance of early awareness. People use it to connect the measuring synaptic, is better than those obtained by the traditional linear method effect. The method has also been used to estimate the maximum information by neurons kindling rate, which can be transfer. The mutual information is shown to use it to evaluate various information of different stimulation produced in neurons in the encoding process value, which is very useful, because people think that stimulate expression is characterized by neuronal complete processing. At the same time, using it to calculate the effectiveness evaluation model of code to model transmission absolute information quantity. Mutual information on the basis of information theory, by measuring the two time variable individual and joint probability density distribution, using the concept of entropy, is to quantify the statistical independence between two time variable. The prevention and elimination of brain fatigue, has become a problem to be solved urgently. Wisdom comes from the brain, not the vitality of the healthy brain, it will be difficult to imagine a superhuman wisdom. China's large and medium-sized city with more than $60 \%$ whitecollar every desk work late into the night, of which $80 \%$ white-collar workers due to the long-term excessive use of the brain with cerebral fatigue in different degrees, among which six into above white-collar workers often dizziness, headache, four into above white-collar lead to neurasthenia, insomnia, forgetfulness and even the decline of brain function, and the two into white collar causing other physical and mental disease, and even some white-collar overwhelmed and Dutch act. According to a sample survey of japanenese showed: mental workers daily average of $59.6 \%$ for a long time when learning or brain brain work a long time, often feel dizziness, focus on it, reducing the efficiency of study and work, this is the brain fatigue performance. How to alleviate the brain fatigue, someone smoking, rest or sleep method to adjust, but smoking would bring about serious side effects to the brain and body health. The expert reminds, relieve brain fatigue is a better method of proper exercise, such as walking, playing exercises, and etc, but the intensity of activity is not too large, the time should not be too long. The brain can be relaxed in the movement, the elimination of fatigue. Especially for those who work pressure, heavy mental burden, work, career, relationships and family and so on, always are a kind of thinking, anxiety, boredom, fear, depression and stress person, more should go through the proper exercise, to relieve brain fatigue, improve sleep quality, avoid mental disorder. Our school for up to ten hours a day, $40.2 \%$ of the students desk study late into the night, only $1 \%$ of people with brain health consciousness. A lot of people have such experience, now the implementation of the burden for students, each lesson 45 minutes after class regulations, let the students brain have proper rest, the implementation of class-break setting-up exercise, which make the brain fatigue recovery. The student is very important for brain health. Brain fatigue long time high load caused by mental activity can cause the decrease of alertness, think slow speed of information processing and cognitive function abate. Therefore, the study of brain fatigue induced changes of EEG has important scientific significance and clinical application value.

\section{Mutual Information Theory}

In probability theory and information theory, the mutual information[9] (sometimes known by the archaic term transinformation) of two random variables is a quantity that 
measures the mutual dependence of the two random variables. The most common unit of measurement of mutual information is the bit, when logarithms to the base 2 are used.

Formally, the mutual information of two discrete random variables $\mathrm{X}$ and $\mathrm{Y}$ can be defined as:

$$
I(X ; Y)=\sum_{y \in Y} \sum_{x \in X} p(x, y) \log \left(\frac{p(x, y)}{p(x) p(y)}\right)
$$

where $\mathrm{p}(\mathrm{x}, \mathrm{y})$ is the joint probability distribution function of $\mathrm{X}$ and $\mathrm{Y}$, and $\mathrm{p}(\mathrm{x})$ and $\mathrm{p}(\mathrm{y})$ are the marginal probability distribution functions of $\mathrm{X}$ and $\mathrm{Y}$ respectively.

In the case of continuous random variables, the summation is replaced by a definite double integral:

$$
I(X ; Y)=\int_{Y} \int_{X} p(x, y) \log \left(\frac{p(x, y)}{p(x) p(y)}\right) d x d y
$$

Intuitively, mutual information measures the information that $\mathrm{X}$ and $\mathrm{Y}$ share: it measures how much knowing one of these variables reduces uncertainty about the other.

For example, if $\mathrm{X}$ and $\mathrm{Y}$ are independent, then knowing $\mathrm{X}$ does not give any information about $\mathrm{Y}$ and vice versa, so their mutual information is zero.

At the other extreme, if $\mathrm{X}$ and $\mathrm{Y}$ are identical then all information conveyed by $\mathrm{X}$ is shared with $\mathrm{Y}$ : knowing $\mathrm{X}$ determines the value of $\mathrm{Y}$ and vice versa. As a result, in the case of identity the mutual information is the same as the uncertainty contained in $\mathrm{Y}$ (or $\mathrm{X}$ ) alone, namely the entropy of $\mathrm{Y}$ (or X: clearly if $\mathrm{X}$ and $\mathrm{Y}$ are identical they have equal entropy).

Mutual information is a measure of the inherent dependence expressed in the joint distribution of $\mathrm{X}$ and $\mathrm{Y}$ relative to the joint distribution of $\mathrm{X}$ and $\mathrm{Y}$ under the assumption of independence. Mutual information therefore measures dependence in the following sense: $\mathrm{I}(\mathrm{X} ; \mathrm{Y})=0$ if and only if $\mathrm{X}$ and $\mathrm{Y}$ are independent random variables. This is easy to see in one direction: if $X$ and $Y$ are independent, then $p(x, y)=p(x) p(y)$, and therefore:

$$
\log \left(\frac{p(x, y)}{p(x) p(y)}\right)=\log 1=0
$$

Moreover, mutual information is nonnegative (i.e. $\mathrm{I}(\mathrm{X} ; \mathrm{Y}) \geq 0$; see below) and symmetric (i.e. $\mathrm{I}(\mathrm{X} ; \mathrm{Y})=\mathrm{I}(\mathrm{Y} ; \mathrm{X}))$.

Mutual information can be equivalently expressed as

$$
\begin{aligned}
I(X ; Y) & =H(X)-H(X \mid Y) \\
& =H(Y)-H(Y \mid X) \\
& =H(X)+H(Y)-H(X, Y) \\
& =H(X, Y)-H(X \mid Y)-H(Y \mid X)
\end{aligned}
$$

where $\mathrm{H}(\mathrm{X})$ and $\mathrm{H}(\mathrm{Y})$ are the marginal entropies, $\mathrm{H}(\mathrm{X} \mid \mathrm{Y})$ and $\mathrm{H}(\mathrm{Y} \mid \mathrm{X})$ are the conditional entropies, and $\mathrm{H}(\mathrm{X}, \mathrm{Y})$ is the joint entropy of $\mathrm{X}$ and $\mathrm{Y}$. Using Jensen's inequality on the definition of mutual information we can show that $\mathrm{I}(\mathrm{X} ; \mathrm{Y})$ is non-negative, consequently, $H(X) \geq H(X \mid Y)$. 
Intuitively, if entropy $\mathrm{H}(\mathrm{X})$ is regarded as a measure of uncertainty about a random variable, then $\mathrm{H}(\mathrm{X} \mid \mathrm{Y})$ is a measure of what $\mathrm{Y}$ does not say about $\mathrm{X}$. This is "the amount of uncertainty remaining about $\mathrm{X}$ after $\mathrm{Y}$ is known", and thus the right side of the first of these equalities can be read as "the amount of uncertainty in $\mathrm{X}$, minus the amount of uncertainty in $\mathrm{X}$ which remains after $\mathrm{Y}$ is known", which is equivalent to "the amount of uncertainty in $\mathrm{X}$ which is removed by knowing $\mathrm{Y}^{\prime}$. This corroborates the intuitive meaning of mutual information as the amount of information (that is, reduction in uncertainty) that knowing either variable provides about the other.

Note that in the discrete case $\mathrm{H}(\mathrm{X} \mid \mathrm{X})=0$ and therefore $\mathrm{H}(\mathrm{X})=\mathrm{I}(\mathrm{X} ; \mathrm{X})$. Thus $\mathrm{I}(\mathrm{X} ; \mathrm{X}) \geq$ $\mathrm{I}(\mathrm{X} ; \mathrm{Y})$, and one can formulate the basic principle that a variable contains at least as much information about itself as any other variable can provide.

Mutual information can also be expressed as a Kullback-Leibler divergence, of the product $\mathrm{p}(\mathrm{x}) \times \mathrm{p}(\mathrm{y})$ of the marginal distributions of the two random variables $\mathrm{X}$ and $\mathrm{Y}$, from $\mathrm{p}(\mathrm{x}, \mathrm{y})$ the random variables' joint distribution:

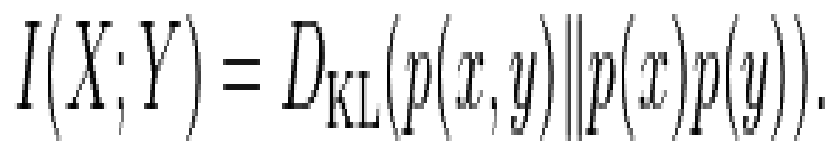

Furthermore, let $\mathrm{p}(\mathrm{x} \mid \mathrm{y})=\mathrm{p}(\mathrm{x}, \mathrm{y}) / \mathrm{p}(\mathrm{y})$. Then

$$
\begin{aligned}
I(X ; Y) & =\sum_{y} p(y) \sum_{x} p(x \mid y) \log _{2} \frac{p(x \mid y)}{p(x)} \\
& =\sum_{y} p(y) D_{\mathrm{KL}}(p(x \mid y) \| p(x)) \\
& =\mathbb{E}_{Y}\left\{D_{\mathrm{KL}}(p(x \mid y) \| p(x))\right\} .
\end{aligned}
$$

Thus mutual information can also be understood as the expectation of the KullbackLeibler divergence of the univariate distribution $\mathrm{p}(\mathrm{x})$ of $\mathrm{X}$ from the conditional distribution $\mathrm{p}(\mathrm{x} \mid \mathrm{y})$ of $\mathrm{X}$ given $\mathrm{Y}$ : the more different the distributions $\mathrm{p}(\mathrm{x} \mid \mathrm{y})$ and $\mathrm{p}(\mathrm{x})$, the greater the information gain.

Sometimes it is useful to express the mutual information of two random variables conditioned on a third.

$$
I(X ; Y \mid Z)=\mathbb{E}_{Z}(I(X ; Y) \mid Z)=\sum_{s \in Z} \sum_{y \in Y} \sum_{x \in X} p_{Z}(z) p_{X, Y}, Z(x, y \mid z) \log \frac{p_{X, Y} \mid Z(x, y \mid z)}{p_{X \mid Z}(x \mid z) p_{Y \mid Z}(y \mid z)^{\prime}}
$$

which can be simplified as

$$
I(X, Y \mid z)=\sum_{s \in Z} \sum_{y \in Y} p_{X, X} p_{X},(x, y, z) \log _{0} \frac{p_{Z}(z) p_{X} Y, Z(x, y, z)}{p_{X, Z}(x, z) p_{Y, Z}(y, z)}
$$

Conditioning on a third random variable may either increase or decrease the mutual information, but it is always true that. 


$$
I(X ; Y \mid Z) \geq 0
$$

for discrete, jointly distributed random variables $\mathrm{X}, \mathrm{Y}, \mathrm{Z}$. This result has been used as a basic building block for proving other inequalities in information theory.

\section{Method}

From the perspective of the information theory, the other containing adjacent electrode signal on the information one electrode, information transmission, that is, mutual information. The transmission of information describes the representative of each lead of the cerebral cortex of the brain regions closely related degree. Select a lead for reference, sum of information exchange to compute the leads and other leads to the comprehensive, as mutual information values of the lead, then followed by selecting the other one lead to calculate the sum of reference lead mutual information with other lead. So you can get different information exchange position lead. The so-called information entropy, is a mathematical whose concept is abstract, might as well put the information entropy is understood as a probability of occurrence of specific information. Generally speaking, when a message appears when the probability is higher, indicating that it was spread more widely, or say, cited higher degree. We can think of, from the perspective of information dissemination, information entropy can express the value of information. So we have a measure of information value standard, can make more inferences about the circulation problem of knowledge. In 1932 Dietch first on EEG were analyzed using Fu Liye transform, since then, the field were introduced into the classical method of time domain analysis, frequency domain analysis of EEG analysis etc. In recent years is as emerged as the time frequency analysis, higher order spectral in EEG analysis using analysis, nonlinear dynamics analysis, artificial neural network analysis and other modern processing technology, new development they represent modern analysis methods of EEG signals.

\subsection{Method of Time Frequency Analysis}

In 1910, Harr proposed a wavelet orthonormal basis thought, the earliest in 1981, Morlet first proposed the concept of "wavelet analysis", and the establishment of the Morlet wavelet named after him. In 1984 Grossman and Morlet proposed a mathematical form of wavelet transform. In 1987, Mallat skillfully combines the multiscale analysis is introduced in wavelet analysis, including the wavelet decomposition and reconstruction of wavelet transform, according to the structure and the signal wavelet function at the same time, Daubechies is constructed with a limited set of orthogonal wavelets, so far, the system of wavelet analysis theory established. Wavelet transform with multi-scale, constant relative bandwidth, proper selection of basic wavelet can have the characterization of the signal's local characteristic in time domain and frequency domain characteristics of the ability. Wavelet transform in scale transformation under different equivalent to a different center frequency band pass filter, so it has been widely used in abnormal wave detection in the EEG signal, such as spike wave, spike and slow wave complex and etc. Senhadji wavelet to detect the non periodic EP signals evoked by stimulation, to identify the extent of brain injury in Thakor by using wavelet analysis, wavelet analysis is an effective method to analyze the findings of brain injury characteristics. Anna Caterina Merzagora for EEG feature extraction based on wavelet transform, to achieve detection to talk to a person's behavior, the results of the study show that, the wavelet coefficients corresponding to a great difference between the liars and the brain waves in the beta band. This shows that the wavelet coefficient will become a new feature for lie detection. Nanaho Tanei wavelet transform between the effective detection of left and right eye behavior and EEG signal connection. 


\subsection{Method of High Frequency Analysis}

In early twentieth Century 60 years, some mathematicians began to study the higher order statistics, however, which was only limited to the theoretical analysis. Until 1989, Vail held its first International Conference on high order spectral analysis, in a move that marked the beginning of a new era in the field of signal processing. In 1990 IEEE Trans. Automatic Control and IEEE Trans, on ASSP had published the monograph about higher order statistics, so far, research on high order statistics has entered a new stage of development. In recent years, higher order statistics as a new signal processing method has received more and more attention of researchers. High order statistics has been widely applied to many fields, including sonar, radar, plasma physics, biomedicine, seismic data processing, image reconstruction, harmonic retrieval, time delay estimation, adaptive filter, array processing, blind deconvolution and blind equalization. As people in-depth study of EEG, EEG found in more cases showed typical nonlinear stochastic process. It adopts the research technique of EEG signals of various signal processing, making the study of EEG signal further development. The traditional power spectrum analysis contains information basically is contained in the autocorrelation function, which for complete statistics of a Gauss process is enough, but can't get there about Gauss of deviation and the nonlinear higher order information, however, with high order cumulant spectrum is defined containing such information. By using the method of high order cumulant of EEG signal biological treatment has the following advantages, which can inhibit the adding Gauss noise, effectively improve the performance of signal parameter estimation; be able to detect and characterize the signal nonlinear characteristics or the identification of nonlinear systems, and can extract random process deviates from the Gauss distribution of the effective degree, detection of signal amplitude information and provide the phase information of the signals, which can be used for the identification of non-minimum phase system. Bispectrum is high order spectral order minimum, higher order statistics method is also the most simple, it contains all the features of the high order spectrum, so it is widely applied,some foreign scholars has done a lot of research on the mathematical theory of double spectrum and its application. A growing body of research suggests that EEG signal is a nonlinear, non-random process of Gauss, the bispectrum analysis method can extract effective conventional spectral analysis methods cannot obtain useful information.

\subsection{Method of Nonlinear Dynamic Analysis}

Nonlinear science arose in twentieth Century 60 in twentieth Century, known as "the third revolution of natural science". As the main performance of nonlinear system is chaotic, non-periodic, non-stochastic, nonlinear, sensitive dependence on initial conditions, and etc. In most physical and biological systems, multi dominated by similar chaotic behavior, the system completely determine the traditional almost does not exist in nature, compared with the traditional linear system description method, the nonlinear system is more suitable to describe the real behavior of nature. The brain is a very complex nonlinear system, the EEG signal is a nonlinear time sequence of a nonstationary, time-varying, complicated. In 1985, Babloyantz first analysis of nonlinear dynamics and chaos theory to the EEG signal, and obtains the sleep EEG signal of human this conclusion is a chaotic signal, which is applied to the nonlinear dynamics method for extraction and analysis of EEG signal, has opened up a new way for the process and features of the further study of brain activity. At present, more commonly used nonlinear dynamical methods mainly include, Lyapunov exponent, correlation dimension and complexity, and etc. The use of different methods, but the main objective is as follows: (1) revealed brain function, mainly study the human body in different physiological states (such as quiet, thinking, anesthesia, sleep), the nonlinear dynamic characteristics in different brain states, to understand the working mechanism of brain function (2) for the 
treatment of brain disease, research this is the main body in the study of pathological conditions (such as epilepsy, Alzheimer's disease, brain injury and so on) are there any changes in nonlinear dynamics, and provide reference for the clinical analysis and diagnosis. In 1965, Kolmogorov firstly gives the definition of complexity measure KC 1976 Lempel and Ziv, to achieve on the Kolmogorov entropy algorithm, called LempelZiv algorithm, this algorithm is used to increase the measurement sequence and the new mode of speed, the 1987 Kasper and Schuster gives the implementation procedure of the algorithm. In 1991, Pincus first proposed and defined a measure of sequence entropy approximation method to measure - complexity (ApEn). In 2000 Richman of the approximate entropy algorithm is improved, proposes the sequence of a better method to measure the degree of complexity of the sample entropy. Complexity is an index in time series, a time sequence through a specific algorithm processing, which can get a complexity value. This value indicates is irregular degree sequences, the irregular change of sequence complexity is higher, and the complexity of the sequence of values is relatively simple as constant sequence, periodic sequence of this model is relatively low. In the complexity analysis of biomedical signals, often need a coarse graining preprocessing of the original signal, the pretreatment may result in loss of information contained in the original signal, power and even in some cases fundamentally changed the original signal the nature.

\section{Result}

According to the research content and purpose, design the corresponding experiment, and get the test quiet EEG data state and fatigue condition. The use of MATLAB simulation experiments, to get mutual information correlation matrix.

Description: the Figure 1 below matlab simulation diagram is the image manifestations of brain electrical mutual information value. The abscissa represents the electrode, color line is based on the color bar right, to said electrode. The ordinate is the mutual information values. The correlation with the mutual information values is more stronger representative electrode. Figure 2 is the representative of the gray image mutual information. The transverse and longitudinal coordinate representing the electrode, gray represents the right size of the mutual information. Longer values mean a stronger influence between the two electrodes. The first experimental quiet condition related to mutual information image is as Figure 1, Mutual information related to image the first experimental quiet state is as Figure 2, The first experimental continuous reading for four hours under the state of fatigue related to mutual information image is as Figure 3, The first experimental continuous reading related to mutual information image four hours under fatigue condition is as Figure 4, The second experiment quiet condition related to mutual information image is as Figure 5, Mutual information related to image second experiment quiet state is as Figure 6, The second experiment continuous reading for two hours under the state of fatigue related to mutual information image is as Figure 7, The second experiment continuous reading related to mutual information image for two hours under the state of fatigue is as Figure 8. 


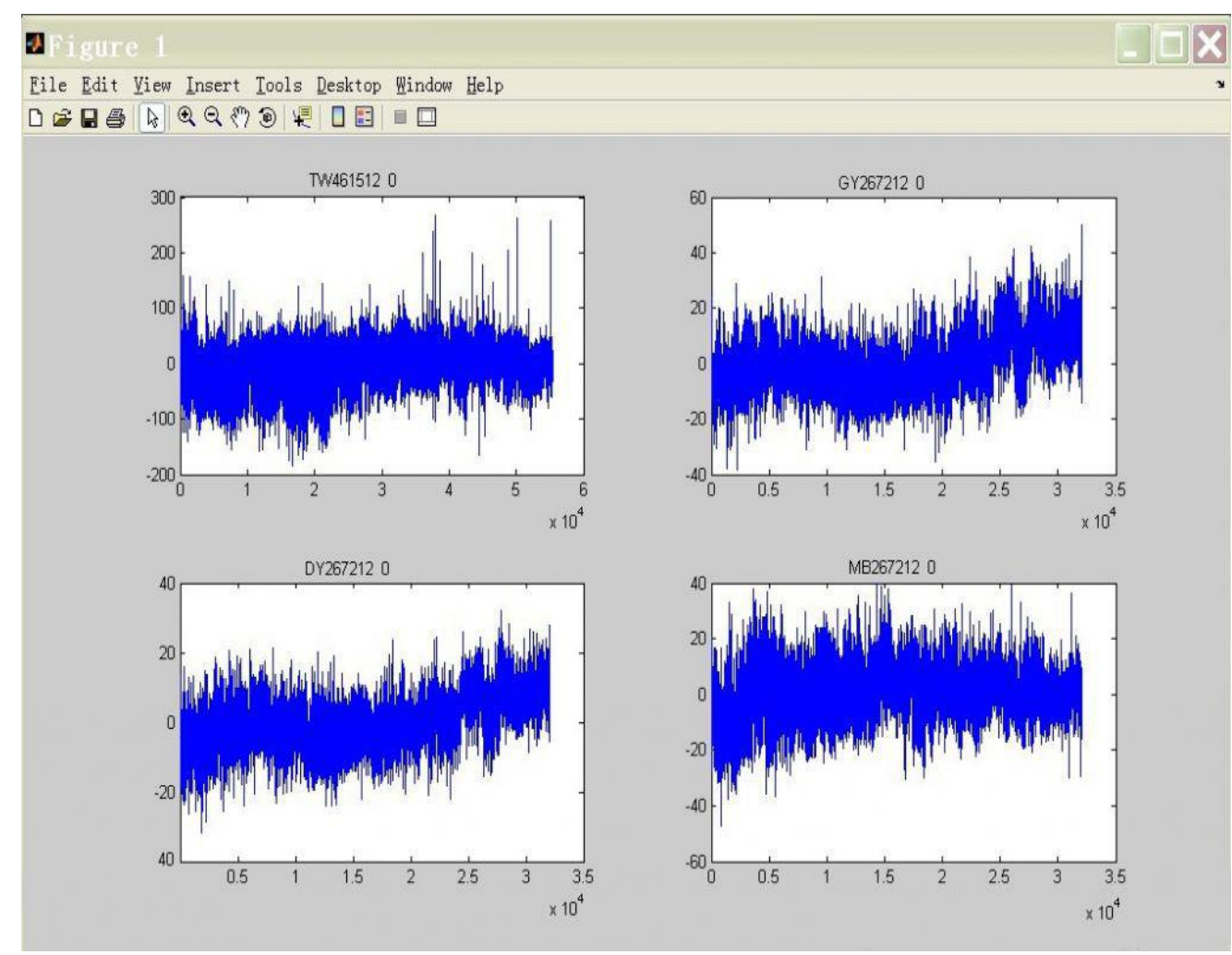

Figure 1. Ordinate of Mutual Information



Figure 2. Mutual Information Values(1)

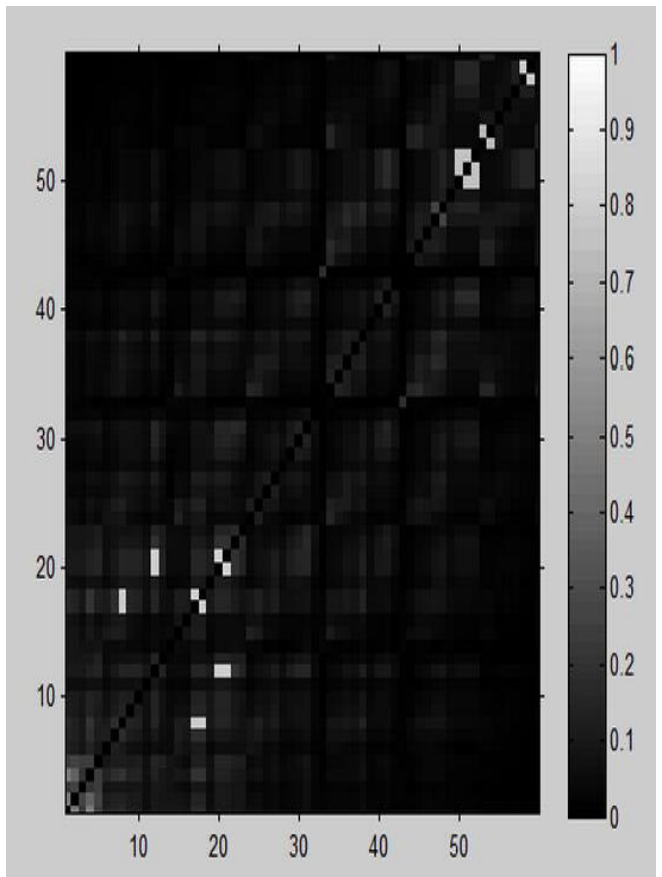

Figure 3. Mutual Information Values (2) 


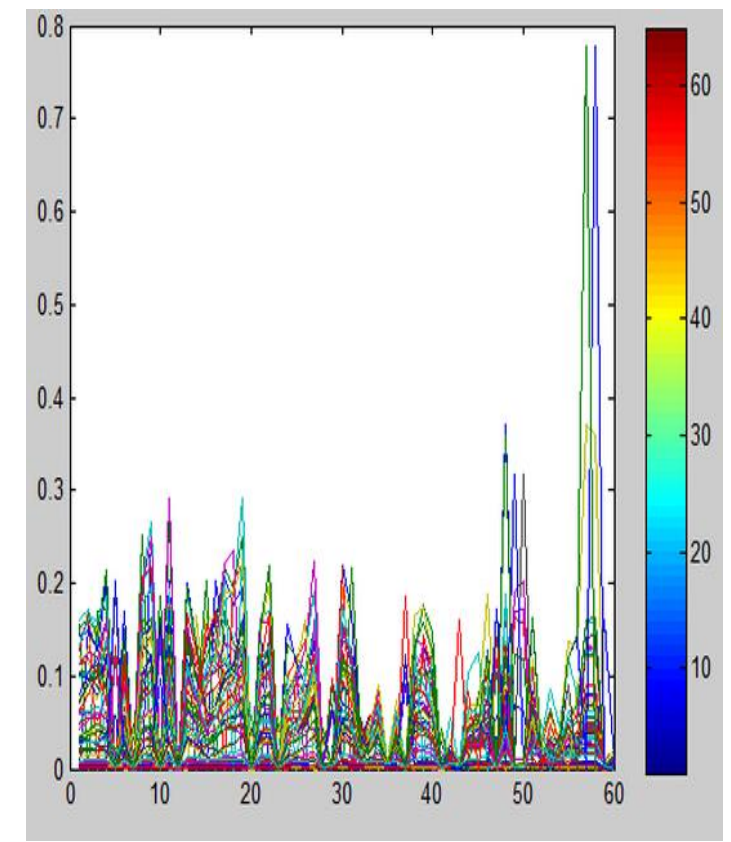

Figure 4. Mutual Information Values (3)



Figure 6. Mutual Information Values (5)

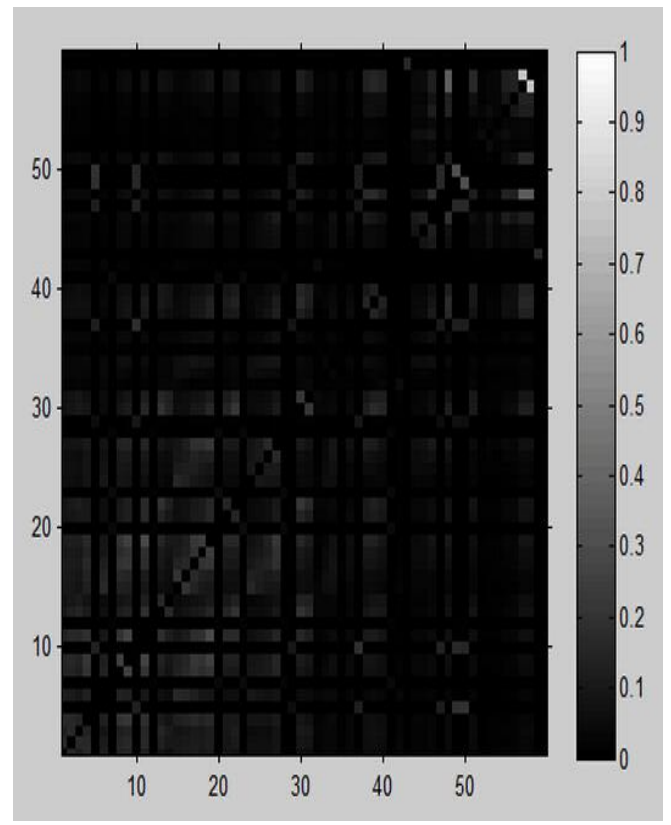

Figure 5. Mutual Information Values (4)

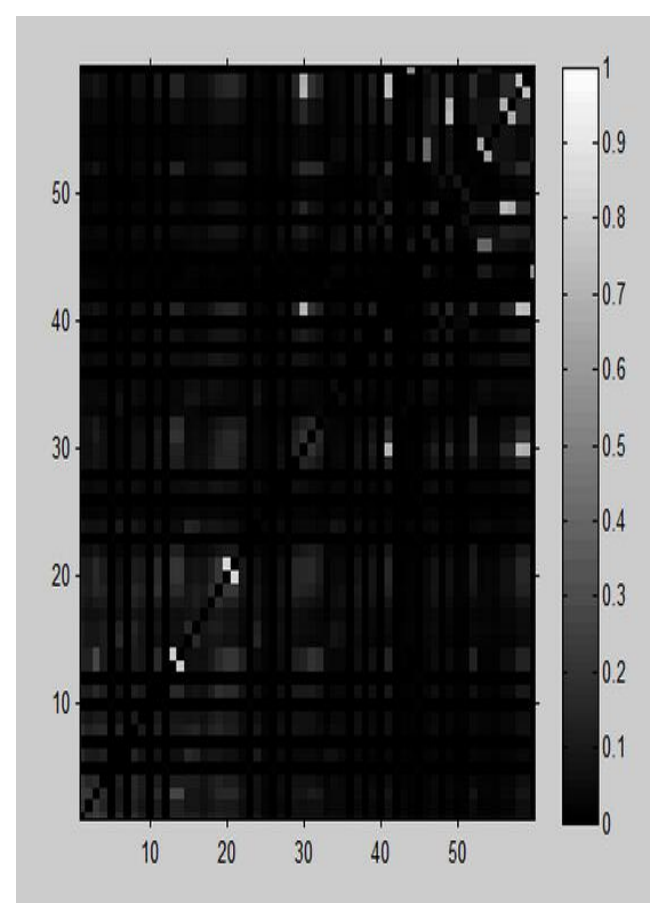

Figure 7. Mutual Information Values (6) 


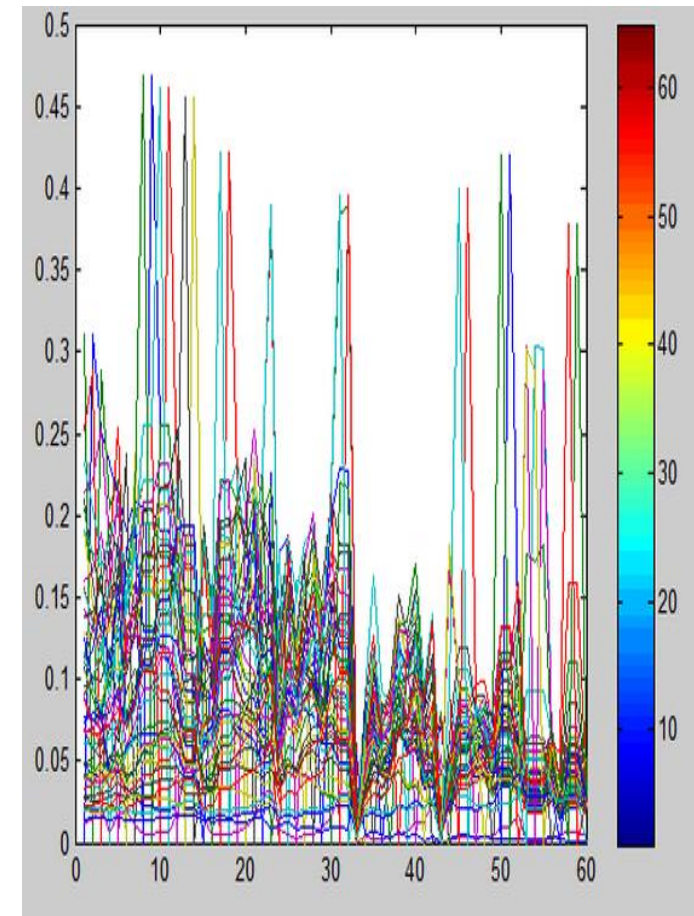

Figure 8. Mutual Information Values (7)

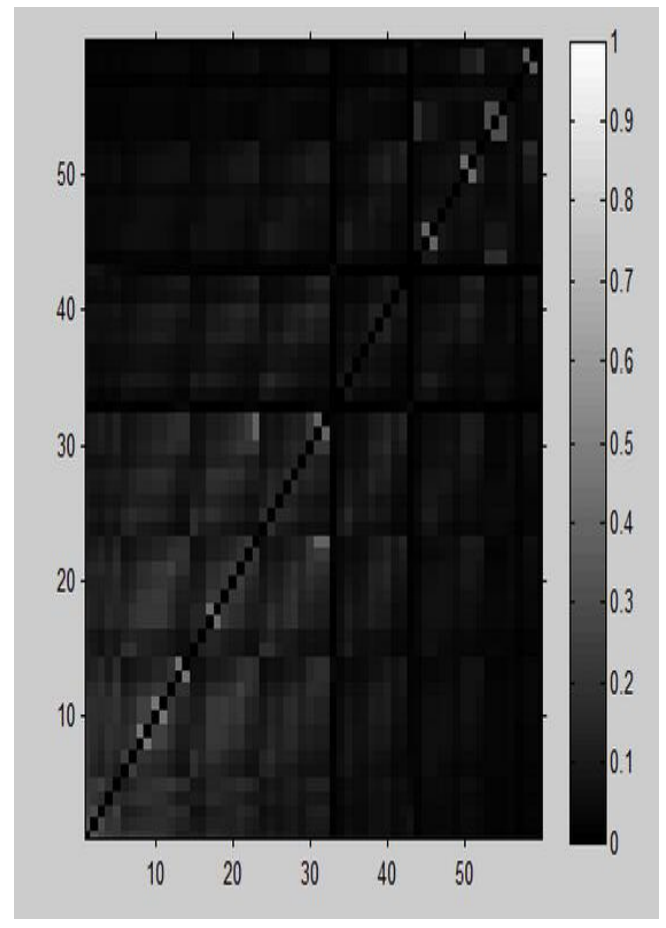

Figure 9. Mutual Information Values (8)

\section{Discussion}

Further study of the data, with analysis of simulation results, is through the joint entropy from information entropy, arbitrary each channel EEG signal was calculated by the two channels of EEG signal and the channel EEG mutual information matrix, the correlation degree analysis of signal of each channel was height, the effect of brain fatigue was studied deeper mutual information feature of EEG

\section{Acknowledgments}

The authors gratefully acknowledge the project Supported by Foundation of He'nan Educational Committee under grant No 14B120002, the project supported by the Scientific Research Starting Foundation for High-level Talents, North China Institute of Water Conservancy and Hydroelectric Power under grant No.201117 and the project supported by the Open Project Foundation of Key Laboratory of Innovation Method and Decision Management System of Guangdong Province under grant No. 2011A060901001-12D. the project supported by the National Natural Science Foundation of China under grant No. U1504622. The authors would like to thank the anonymous reviewers and the editors for their valuable comments and suggestions.

\section{References}

[1] Y. Zhou, C. Zhang and X. Zhou, "Study on EEG Password Based on Brain Friend Mode", IJACT: International Journal of Advancements in Computing Technology, vol. 5, no. 1, (2013), pp. 733- 741.

[2] Y. Zhou, "Study on Game Theory and Model of ERP based on EEG", International Journal of Signal Processing, Image Processing and Pattern Recognition, vol. 7, no. 4, (2014), pp.127-136.

[3] D. Jiang, "Study on auxiliary game platform based on PC and PDA", AISS, vol. 3, no.7, (2011), pp. 287-295.

[4] Y. Zhou, J. Li and X. Zhou, "Study on EEG Password based on AR Power Spectrum and BP network", JCIT: Journal of Convergence Information Technology, vol. 7, no. 9, (2012), pp. 6-14.

[5] D. Jiang, "Study on driving system based on EEG", Applied Mechanics and Materials, vol.63, no.1, (2011), pp. 579-582. 
[6] D. Jiang, J. Yin and J. Hu, "Study on arithmetic library of signal process based on PDA", Advanced Materials Research, vol.282, no.1, (2011), pp. 326-329.

[7] X. Li and D. Jiang, "Study on the application of COM method in motor imagine", Advanced Materials Research, vol.187, no.1, pp.762-766, (2011).

[8] Y. Zhou, "Study on Brain Matching Based on Mobile Platform", International Journal of Signal Processing, Image Processing and Pattern Recognition, vol. 8, no. 12, (2015), pp.1-8.

[9] Cilibrasi and R. Paul Vitányi, "Clustering by compression", IEEE Transactions on Information Theory, vol. 51, no. 4, (2005), pp. 1523-1545.

\section{Author}

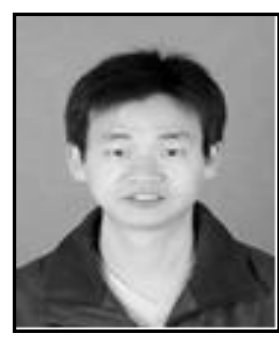

Yu Zhou, he was born in China. He received his B.E. in Automation from Henan University in 2004, China, his M.E. in Detection Technology and Automatic Equipment from Guangdong University of Technology in 2007, China, and his Ph.D. in Control Theory and Control Engineering from China University of Mining and Technology (Beijing) in 2010. He has been a visiting Ph.D. student at Department of Electrical and Computer Engineering, University of Alberta, Canada from Sep. 2009 to Sep. 2010. He is now working at North China University of Water Conservancy and Hydroelectric Power as a lecturer. As a researcher, he also works at Key Laboratory of Innovation Method and Decision Management System of Guangdong Province, Guangzhou. His current research interests include the following areas: artificial neural network, pattern recognition, intelligence computing and intelligent control. 
International Journal of Signal Processing, Image Processing and Pattern Recognition Vol. 10, No. 5 (2017) 